\title{
A model of developmental time applied to planktonic embryos
}

\author{
Dag L. Aksnes ${ }^{1, *}$, Christofer Troedsson ${ }^{2}$, Eric M. Thompson ${ }^{2}$ \\ ${ }^{1}$ Department of Biology, University of Bergen, PO Box 7800, 5020 Bergen, Norway \\ ${ }^{2}$ Sars International Centre for Marine Molecular Biology, Thormøhlensgate 55, 5008 Bergen, Norway
}

\begin{abstract}
Planktonic embryos are unprotected and experience high mortalities. Risk exposure and its consequences for survival depend very much on developmental time, which, in turn, is strongly affected by temperature. Developmental times in planktonic embryos have recently been correlated with cell cycle duration and egg size. Here, we elaborate on this by deriving a developmental time model from cell proliferation dynamics in which the effect of temperature on developmental time is modelled through the effect of temperature on cell cycle duration. Published cell lineage data from Caenorhabditis elegans and on developmental times in planktonic embryos were used to assess the validity of model assumptions. Analyses demonstrate that a common parameter set accounts for 94 to $95 \%$ of observed variance in developmental times for species from the same phylum. This percentage drops considerably when species from different phyla are included in a single analysis, suggesting taxonomic differentiation in the developmental parameters of the model. The model provides a theoretical framework for anchoring observed variability in organism traits at the cellular scale, and we provide suggestions for future experimental work designed to connect developmental time in organisms to their cell proliferation dynamics.
\end{abstract}

KEY WORDS: Development $\cdot$ Age $\cdot$ Cell cycle duration $\cdot$ Temperature $\cdot$ Plankton Resale or republication not permitted without written consent of the publisher

\section{INTRODUCTION}

Planktonic embryos are among the least protected and most vulnerable marine embryos (Staver \& Strathmann 2002). Average embryonic mortality rates of Calanus finmarchicus in the Norwegian Sea amount to $82.8 \% \mathrm{~d}^{-1}$, suggesting that only $2.7 \%$ of the eggs survive to the first larval stage (Ohman \& Hirche 2001). Due to investments such as the development of resistance to predators, mortality risk decreases as plankton develop from eggs to adults. Developmental time is therefore a critical factor determining risk exposure at different ontogenetic stages. Slower development generally translates to longer exposure and higher risk. Strathmann et al. (2002) showed that planktonic embryos with short developmental times prior to first swimming tend to be strongly selected, and that this selection is facilitated by short cell cycle durations. In a multiple regression, they found that about $50 \%$ of the variance in developmental times of species from different phyla could be accounted for by the cell cycle duration from the 2- to 4-cell stage, together with the size of the egg. Here, we derive a model that mechanistically connects developmental time with cell cycle duration and other aspects of cell proliferation dynamics.

Temperature affects developmental time, especially for non-feeding stages, but also for later planktonic stages (Campbell et al. 2001, Gillooly et al. 2002, Staver \& Strathmann 2002). The way organisms respond to changes in temperature is often parameterised with a temperature sensitivity formulation such as the $Q_{10}$-relationship. Gillooly et al. (2002) found that the central tendency of the temperature dependencies of developmental time for many different groups of organisms was close to a quantity predicted from the activation energy for metabolic reactions. This fact explained a large part of the between-species variation in temperature sensitivity. 
Nonetheless, individual species do deviate from the central tendency, and Staver \& Strathmann (2002) found that the $Q_{10}$ values for time to first swimming in planktonic embryos ranged from about 2 to 4 . Temperature affects cell division clocks (Schibler 2003), and in our model we hypothesise that the temperature sensitivity of the developmental time of the whole organism is equal to that of the cell cycle duration. We assess whether the assumptions of our model are consistent with development in Caenorhabditis elegans, for which a detailed data set exists linking cell fate and developmental stage (Sulston \& Horvitz 1977, Sulston et al. 1983), and for a number of planktonic embryos (Staver \& Strathmann 2002). We discuss to what extent our model accounts for observed variability in developmental time in planktonic embryos, and argue that our model will serve as a theoretical framework for future experiments exploring causal factors for variation in developmental time. As shown elsewhere (Aksnes et al. 2006, this volume), this model also serves as a basis for modelling life history such that it integrates developmental clocking, growth and environmental forcing.

\section{MATERIALS AND METHODS}

Model derivation. We describe the developmental stage of an organism by its cell number (symbols are further explained in Table 1). Cell number $\left(N_{j}\right)$ can be expressed as a function of rounds of cell cycle progression:

$$
N_{j}=c \mathrm{e}^{g j}
$$

where $c=1$ cell is the first cell (the fertilised egg), and $j$ counts successive cell cycle rounds. We see that $N_{j}=$ $1,2,4, \ldots$ cells for $j=0,1,2, \ldots$ when $g=\ln 2$ per cycle, which amounts to binary fission without apoptosis and cell cycle exit. The parameter $g$ specifies the increase in cell number for each $j$-cycle. Note that $g$ and $j$ are not expressed in units of time. Generally, we define $g=$ $r-d$, where $r$ and $d$ express cell proliferation and apoptosis per cell cycle, respectively. For binary fission, $g$ takes a maximal value of $\ln 2$ per cycle and is, together with $d$, under cell-lineage-specific regulation. As organs develop, increasing numbers of cells reach their final differentiated state and $g$ decreases, thus being a function of $j$. We denote the first cell cycle duration (from 1 to 2 cells) as $l_{0}$, the second (from 2 to 4 cells) as $l_{1}$, etc. The developmental time $\left(t_{i}\right)$ for Stage $i$, which corresponds to the age of that stage, is defined as the sum of all cell cycle durations starting with $j=0$ and ending with $j=i-1$ :

$$
t_{i}=\sum_{j=0}^{i-1} l_{j}
$$

Most organisms lose synchrony of cell divisions early on, and as the organism develops we must assume that $l_{j}$ represents the average length for 1 cell cycle round.

Studies on embryonic cell lineages in Caenorhabditis elegans (Sulston et al. 1983) show that the average length for cell cycle rounds tends to be longer for successive cell cycles. This may reflect increased time requirements with increasing organism complexity, and we have assumed that average length of cell cycling can be described as a function of normalised cell number according to a power function: $l_{j}=l_{0} n_{j}{ }^{p}$, where $p$ is a scaling coefficient, and $n_{j}$ is normalised cell number $\left(n_{j}\right.$ is used for the sake of dimensional consistency). Here, $n_{j}=N_{j} / c=\mathrm{e}^{g j}$ (see Eq. 1), which has the same numerical value as the cell number $\left(N_{j}\right)$, since $C=1$ cell. We can now write $l_{j}=l_{0} n_{j}{ }^{p}=l_{0} \mathrm{e}^{g i p}$. Substitution into Eq. (2) and use of the formula for the sum of a geometric progression, which requires that the product $x=p g$ (see Table 1) is constant, yields:

$$
t_{i}=\sum_{j=0}^{i-1} l_{j}=\sum_{j=0}^{i-1} l_{0}\left(\mathrm{e}^{x}\right)^{j}=l_{0} \frac{\mathrm{e}^{x i}-1}{\mathrm{e}^{x}-1}
$$

Cell cycle duration is strongly influenced by temperature (Staver \& Strathmann 2002, Schibler 2003), and we express the temperature sensitivity of cell cycle duration as $l_{0}=l \mathrm{e}^{-a T}$, where $a$ is temperature sensitivity, $T$ is temperature $\left({ }^{\circ} \mathrm{C}\right)$ and $l$ is the first cell cycle duration at a reference temperature defined as $0^{\circ} \mathrm{C}$ for mathematical convenience. The parameter a may here represent the common empirical $Q_{10}$ parameter (then $a=\ln Q_{10} / 10$ ) or the more fundamental Boltzmann representation $\left(a=-\alpha /\left(1+T / T_{0}\right)\right.$, see Gillooly et al. 2002 for detailed treatment of the 2 different temperature-sensitivity descriptions, and for definition of terms). Inclusion of temperature in Eq. (3) yields:

$$
t_{i}=l \mathrm{e}^{-a T} \frac{\mathrm{e}^{\mathrm{xi}}-1}{\mathrm{e}^{\mathrm{x}}-1}=b \mathrm{e}^{\mathrm{xi}-a T}-b \mathrm{e}^{-a T}
$$

where the reference timescale $b=1 /\left(\mathrm{e}^{\mathrm{x}}-1\right)$. The way temperature is accounted for implies that the temperature sensitivity of the developmental time of the whole organism is equal to that of the cell cycle duration, and that the temperature sensitivities of all cell cycle durations are identical. This does not need to be valid for true organisms, but serves here as a working frame.

Eq. (4a) can also be written explicitly as a function of normalised cell number, $n$, instead of Developmental Stage $i$ :

$$
t_{i}=b \mathrm{e}^{-a T} n_{i}{ }^{p}-b \mathrm{e}^{-a T}
$$

When the normalised cell number $\left(n_{i}=\mathrm{e}^{g i}\right)$ increases with increasing developmental stage, $i$, the last term of the equation can be ignored:

$$
t_{i} \approx b \mathrm{e}^{\mathrm{xi}-a T}=b \mathrm{e}^{-a T} n_{i}^{p}
$$


Table 1. Symbols and their units used in the derivation of the model and in the parameter estimation. d.l.: a dimensionless quantity

\begin{tabular}{|c|c|c|}
\hline Symbol & Unit & Description \\
\hline a & ${ }^{\circ} \mathrm{C}^{-1}$ & Temperature sensitivity (the model assumes $a=a_{l}=a_{t}$ ) \\
\hline$a_{1}$ & ${ }^{\circ} \mathrm{C}^{-1}$ & Temperature sensitivity of cell cycle duration \\
\hline$a_{t}$ & ${ }^{\circ} \mathrm{C}^{-1}$ & Temperature sensitivity of developmental time \\
\hline$b$ & $\mathrm{~h}$ & A reference time scale given by $b=1 /\left(\mathrm{e}^{x}-1\right)$ \\
\hline C & cell & One cell (the egg) \\
\hline$d$ & cycle $^{-1}$ & Cell number decrease (apoptosis) per cell cycle \\
\hline$D$ & $\mu \mathrm{m}$ & Diameter of the egg \\
\hline$g$ & cycle $^{-1}$ & Cell number increase per cell cycle, $g=r-d$ \\
\hline$i$ & cycle & Development stage corresponding to a cell cycle number \\
\hline$j$ & cycle & Cell cycle number \\
\hline$k$ & cycle $\mu \mathrm{m}^{-1}$ & $\begin{array}{l}\text { Expresses how number of embryonic cell cycles relates } \\
\text { to egg size }\end{array}$ \\
\hline 1 & $\mathrm{~h}$ & First cell cycle duration at a reference temperature $\left(0^{\circ} \mathrm{C}\right)$ \\
\hline$l_{j}$ & $\mathrm{~h}$ & $\begin{array}{l}\text { Cell cycle duration for cell cycle } j \text {, where } j=0 \text { is the first } \\
\text { cycle }\end{array}$ \\
\hline$N_{j}$ & cells & Number of cells at cycle $j$ \\
\hline$n_{j}$ & d.l. & Normalised cell number $\left(=N_{j} / C\right)$ \\
\hline$p$ & d.l. & Scaling coefficient \\
\hline$r$ & cycle $^{-1}$ & Cell proliferation per cell cycle \\
\hline$t_{i}$ & $\mathrm{~h}$ & Age of Developmental Stage $i$ (developmental time) \\
\hline$T$ & ${ }^{\circ} \mathrm{C}$ & Temperature \\
\hline$x$ & cycle $^{-1}$ & $\begin{array}{l}\text { Age expansion coefficient, } x=g p \text {, expressing how age } \\
\text { increases with developmental progression }\end{array}$ \\
\hline
\end{tabular}

described according to a power function of normalised cell number.

Application of the model to data on development in planktonic embryos. Staver \& Strathmann (2002) measured the cell cycle duration from the 2- to 4 -cell stage in planktonic embryos of 33 different species in 10 phyla at 2 different temperatures (Table 1 in Staver \& Strathmann 2002). In our mathematical description, this represents the second cell cycle duration. Experimentally this duration is far better defined than the first. They also measured egg sizes of the different species. Egg size is not an explicit parameter of our model, but egg size reflects the mass and energy available for embryonic development. In the application of their data we, therefore, assume that the size (i.e. cell numbers) of the different planktonic embryos at first swimming relates to the egg size (volume) so that $N_{i} \propto D^{3}$, where $D$ is the measured diameter of the egg. As

Extensive cell-lineage studies on the nematode Caenorhabditis elegans (Sulston \& Horvitz 1977, Sulston et al. 1983) enable us to test whether the $p$ - and $g$ parameters, and thereby $x=p g$, can be considered constant as required for the step from Eq. (2) to Eq. (3). Furthermore, the validity of the assumption that the average length of cell cycle rounds can be described according to a power function of normalised cell number, $l_{j}=l_{0} n^{p}$, can be assessed from the observations of developmental time versus normalised cell number. The expectation is that developmental time also should distribute according to $t \propto n^{p}$ (Eq. $4 \mathrm{~b}$ ).

We obtained the following fit of Eq. (1) to the Caenorhabditis elegans data: $N=1.13 \mathrm{e}^{0.65 i}\left(\mathrm{r}^{2}=0.99\right.$, $\mathrm{p}<0.001)$ and $N=192 \mathrm{e}^{0.14 \mathrm{i}}\left(\mathrm{r}^{2}=0.95, \mathrm{p}<0.001\right)$ for the embryonic and post-embryonic periods, respectively (Fig. 1A). This suggests $g$-estimates of $0.65 \pm 0.02$ and $0.14 \pm 0.03$ cycle $^{-1}( \pm 95 \% \mathrm{CI})$ for the 2 periods, respectively. For the expectation $t \propto n^{p}$ we obtained $t=11.70 n^{0.55}\left(\mathrm{r}^{2}=0.97, \mathrm{p}<0.001\right)$ and $t=7.68 n^{0.79}\left(\mathrm{r}^{2}=\right.$ $0.99, \mathrm{p}<0.001)$ for the embryonic and post-embryonic periods (Fig. 1B), suggesting $p$-estimates of $0.55 \pm 0.07$ and $0.79 \pm 0.07$, respectively. From this we conclude that $p, g$ and $x=p g$ clearly change from the embryonic to the post-embryonic period, but for practical purposes can be considered constant within the 2 periods. The non-random residuals of the regressions, however, should be noted. Furthermore, within the 2 distinct developmental periods it seems justified to consider that the average length of cell cycle rounds can be can be seen from Eq. (1) this corresponds to $i=k \ln D$, where $k$ is a constant. We modified Eq. (4a) in order to make use of the measurements of Staver \& Strathmann (2002):

$$
t=l_{1} \mathrm{e}^{-a(T-10)} \frac{\mathrm{e}^{\mathrm{xk} \ln D}-1}{\mathrm{e}^{\mathrm{X}}-1}
$$

In a single, non-linear, least-squares analysis we estimated the parameters $a, x$ and $k$ from the measurements of egg size $(D)$, cell cycle duration from the 2 - to 4 -cell stage $\left(l_{1}\right.$, measured at $\left.10^{\circ} \mathrm{C}\right)$ and developmental time $(t)$ measured at the temperatures $(T) 10$ and $14^{\circ} \mathrm{C}$ for the 33 species listed in Table 1 in Staver \& Strathmann (2002). Estimations were also made for taxonomic subsets.

Based on these data we also estimated the temperature-sensitivity parameter of cell cycle duration $\left(a_{1}\right)$ for each species according to the formula $a_{1}=\ln \left(l_{1}\left(T_{2}\right) / l_{1}\left(T_{1}\right)\right) /\left(T_{2}-T_{1}\right)$. Here, $l_{1}$ represents the measured cell cycle duration from the 2- to 4-cell stage, and $T_{1}$ and $T_{2}$, the 2 temperatures applied in the experiments. In the same way, we estimated the temperature-sensitivity parameter, $a_{t}$, for the developmental time defined as the time to first swimming. The accuracy of the temperature in the experiments was reported to be $\pm 0.3^{\circ} \mathrm{C}$. Potentially this introduces uncertainty ranges of 0.043 to 0.059 and 0.130 to 0.176 for parametric $a$-values of 0.05 and 0.15 , respectively. The observed effect of temperature on developmental time $\left(a_{t}\right)$ should, according to our model, be equal to the temperature sensitivity of the cell cycle duration 

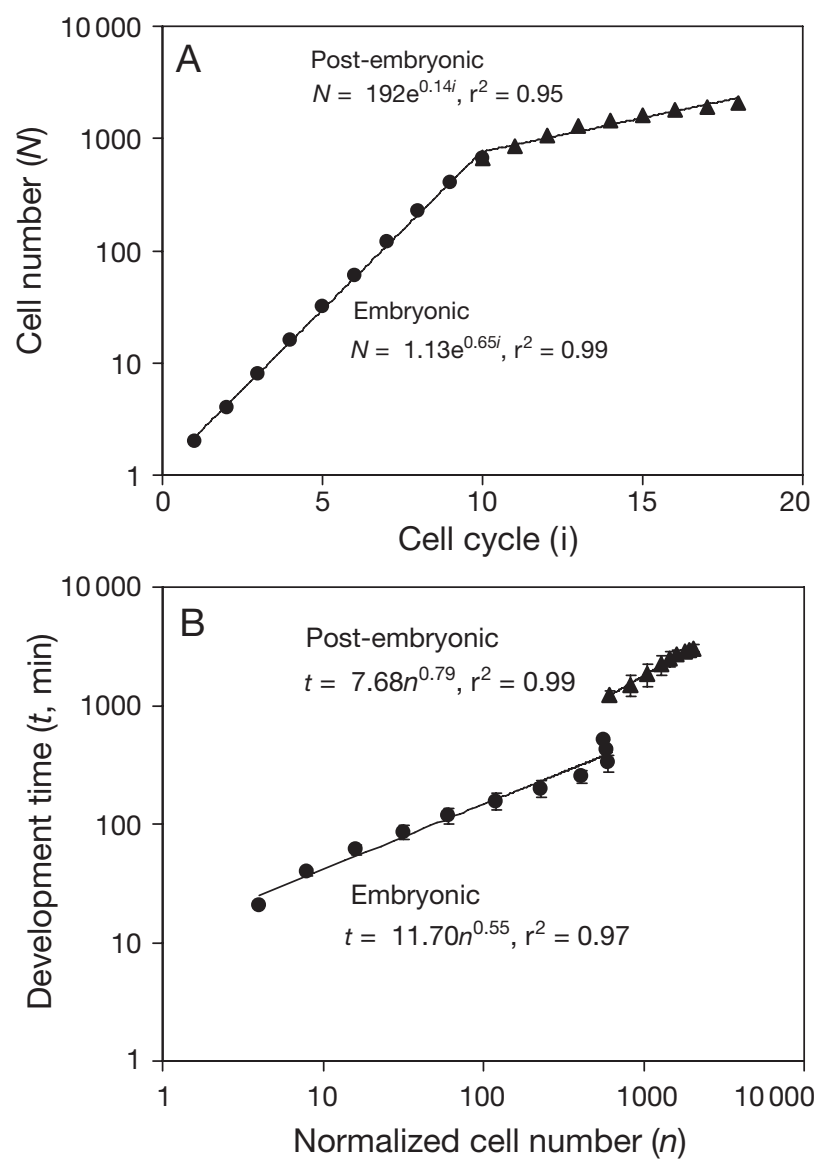

Fig. 1. Caenorhabditis elegans. Development based on data in Sulston \& Horvitz (1977) and Sulston et al. (1983). (A) Eq. (1) is fitted to the observed cell number versus cell cycle. The exponent of the fitted functions is an estimate of the increase in cell number per cell cycle $(g)$. (B) A power function is fitted to observed developmental time versus cell number. Here, the exponent is an estimate of the $p$-coefficient. ( $\bullet$ Embyonic development; $(\mathbf{\Lambda})$ post-embryonic development

$\left(a_{1}\right)$. This further implies that the observed betweenspecies variation in $a_{t}$ should be equal to the betweenspecies variation in $a_{1}$.

\section{RESULTS}

The estimates of $a, x$ and $k$ obtained by non-linear estimation (Eq. 5) using the entire data set of Staver \& Strathmann (2002) accounted for $77 \%$ of the observed variation in developmental time (Table 2). The p-values associated with all 3 estimates were significant (Table 2). The phyla Mollusca and Echinodermata represented the taxonomic subgroups that included most species in the data set. Separate estimation for these 2 groups accounted for 94 and $95 \%$ of the observed variance in developmental time, respectively (Table 2).
Again the associated p-values were significant (Table 2). By excluding Echinodermata and Mollusca, 15 species representing 8 phyla (Cnidaria, Ctenophora, Nemertea, Annelida, Phoronida, Brachipoda, Bryozoa and Urochordata) remained in the data set. For this heterogeneous group only $11 \%$ of the variance in observed developmental times was accounted for by assuming invariant parameter values. None of the p-values were significant at the $5 \%$ level (Table 2).

The temperature sensitivity of cell cycle duration for the different species $\left(a_{1}\right)$ ranged from 0.05 to 0.15 , with a mean of $0.1085 \pm 0.0045( \pm \mathrm{SE}, \mathrm{n}=28)$ (Table 3$)$. The temperature sensitivity of time to swimming $\left(a_{t}\right)$ ranged from 0.06 to 0.15 , with a mean of $0.1076 \pm 0.0058$ ( $\mathrm{n}=$ 28). According to $t$ - and $F$-tests both the means and variances of $a_{l}$ and $a_{t}$ were equal, which is consistent with the hypothesis that the temperature sensitivities of developmental time and cell cycle duration are equal. We also made a regression between $a_{t}$ and $a_{1}$. This was highly significant $\left(\mathrm{r}^{2}=0.42, \mathrm{p}=2 \times 10^{-4}, \mathrm{n}=28\right)$ but $a_{l}$ could only explain $42 \%$ of the variance in $a_{t}$. The residual variance $\left(\mathrm{MS}=3.45 \times 10^{-4}\right.$ ) corresponds to a coefficient of variation in the a-estimates of about $18 \%$. The calculated uncertainty ranges, originating from the reported temperature accuracy of $\pm 0.3^{\circ} \mathrm{C}$ in the experiments (Staver \& Strathmann 2002), corresponds to about $\pm 15 \%$ (see 'Materials and methods'), and this experimental error is potentially responsible for a substantial part of the residual variance.

\section{DISCUSSION}

Staver \& Strathmann (2002) found that the time to first swimming correlated significantly with cell cycle durations $\left(p=0.006\right.$ and $p=0.002$ for 10 and $14^{\circ} \mathrm{C}$, respectively, their Table 2 ). Though only part of the variance $(25 \%)$ in developmental time could be attributed to cell cycle duration, they found that another $25 \%$ could be accounted for by inclusion of egg size additionally to cell cycle duration in a multiple regression analysis. They rejected the possibility that egg diameter correlated with time to first swimming, because larger eggs take longer to divide. Of factors not accounted for in their experiments, Staver \& Strathmann (2002) discussed both cell numbers and the number of cell cycles as possible sources for variance in their observed developmental times. These factors are explicitly expressed in our model, and the egg size measurements were included in our analysis by the assertion that the number of embryonic cell cycles correlates with egg size (i.e. $i=$ $k \ln D$ ). Assuming invariant parameter values $\left(a_{1} x\right.$ and $k$ ) between species, our model accounted for $77 \%$ of the observed variance in the data set cover- 
Table 2. Estimates obtained by fitting Eq. (5) to the experimental data on developmental times in planktonic embryos (Table 1 in Staver \& Strathmann 2002)

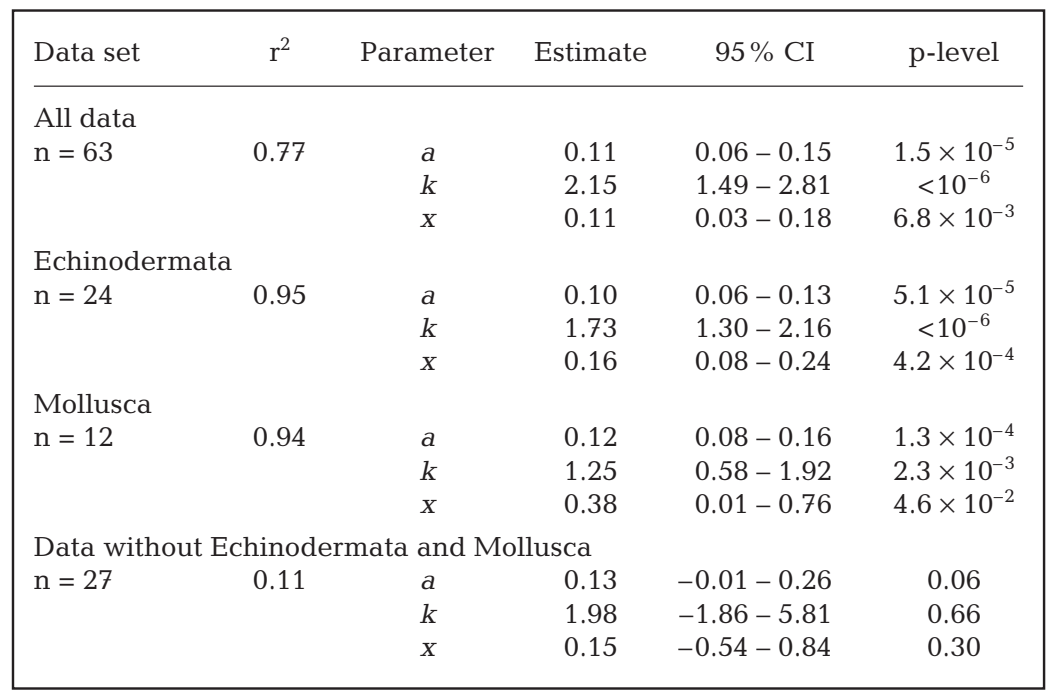

Table 3. Temperature sensitivities of cell cycle duration and developmental time estimated from the measurements given in Table 1 of Staver \& Strathmann (2002) on different species of planktonic embryos. CV: coefficient of variation

\begin{tabular}{|lccccc|}
\hline Description & Symbol & $\begin{array}{c}\text { Range } \\
(\mathrm{h})\end{array}$ & $\begin{array}{c}\text { Mean }( \pm \mathrm{SE}) \\
(\mathrm{h})\end{array}$ & $\begin{array}{c}\mathrm{CV} \\
(\%)\end{array}$ & $\mathrm{n}$ \\
\hline $\begin{array}{c}\text { Temperature sensitivity } \\
\text { of cell cycle duration } \\
\begin{array}{c}\text { Temperature sensitivity } \\
\text { of developmental time }\end{array}\end{array}$ & $a_{1}$ & $0.05-0.15$ & $0.1085 \pm 0.0045$ & 22.1 & 28 \\
\hline
\end{tabular}

gests that both the mean and the variance of $a_{t}$ are equal to those of $a_{l}$ (Table 3), but this is not sufficient to verify the assumption about equality. This implies $100 \%$ correlation between $a_{t}$ and $a_{l}$, while $a_{1}$ explained only $42 \%$ of the variance in $a_{t}$. Although the remaining variance is potentially accounted for by experimental variation in temperature, new experiments are needed to test to what extent the assumption about equal temperature sensitivities between whole organism and cell cycle duration is valid.

Gillooly et al. (2002) derived a general model, based on the first principles of allometry and biochemical kinetics, which predict developmental time as a function of body mass and temperature. The parameters of their model also relate to cellular properties such as the average mass of a cell, the energy needed to create a cell and the biochemical reaction rates for cellular metabolism. Our model is derived from a different set of cellular properties, including number of cell cycles and cell cycle durations, and we use cell number as an index for developmental stage. Our representation of developmental time is complementary to that of Gillooly et al. (2002), extending the

ing all species from the 10 phyla. For each of the 2 taxonomic subgroups, Echinodermata and Mollusca, 94 to $95 \%$ of the variance could be accounted for. On the other hand, for the remaining heterogeneous group covering relatively few species from 8 different phyla, $\leq 11 \%$ of the observed variance in developmental time was accounted for by common parameter values (Table 2). This suggests that different taxonomic groups are characterised by different parameter values, but this needs to be investigated more thoroughly in data sets in which the developmental stages are characterised more accurately by the cell number and/or average number of cell cycle rounds. In the present study, the uncertainties of the parameter estimates, even for the Echinodermata and Mollusca, were too large to reveal significant differences in the parameter estimates of the 2 phyla (see Table 2).

A critical assumption in our model is the hypothesis that the temperature sensitivity $\left(a_{t}\right)$ of the developmental time of the whole organism is equal to that $\left(a_{1}\right)$ of a single cell cycle duration. Our analysis sug- analytical tools available for investigating variability in developmental time within and between species. Cell cycle duration is a distinct biological property that can be precisely measured. If whole-organism developmental time and its temperature sensitivity can be linked to cell cycle duration such as hypothesised here, this will be a useful experimental tool. Measuring the temperature sensitivity of cell cycle duration will generally be easier and more practical than detailing whole-organism developmental time with attendant temperature sensitivity. We do not suggest that this eliminates the need for measurements on whole organisms, but rather argue for the experimental approach taken by Strathmann et al. (2002) and Staver \& Strathmann (2002) in their search for sources of variability in organism traits at the cellular scale. Our model should be a useful tool in assessing such variability, and it provides a theoretical framework for interpretation and integration of measurements of cell cycling characteristics with quantitative models of developmental time. We believe that 2 categories of future experiments are useful to eluci- 
date factors determining developmental time. First, experimentation is needed to see to what extent the temperature sensitivity of developmental time corresponds to that of cycle duration of single cells. This requires a high degree of temperature control in order to minimise the errors of the $a_{t^{-}}$and $a_{l^{-}}$-estimates. Second, experimental comparisons between different species and taxonomic groups, as well as within species, will reveal to what extent temperature sensitivities (a), the scaling parameter $p$ and the cell growth parameter $g$ vary between and within species. Such experimentation will not only provide insight into the life-history constraints of planktonic organisms, but should also address questions about universality in scaling relations in a broader sense.

Acknowledgements. We thank D. Chourrout, Ø. Fiksen, N. Finn, J. Giske, C. Jørgensen, M. Mangel, J. Nejstgaard, A. Wargelius and several anonymous reviewers for comments and discussions. This study was supported by EUROGEL (EVK3-CT-2002-00074) and the Grants 169601 (D.L.A.) and 145326/432 (E.M.T) from the Norwegian Research Council. M. D. Ohman and the California Current Ecosystem LTER site are acknowledged for support during a sabbatical year (D.L.A.) at Scripps Institution of Oceanography.

Editorial responsibility: Otto Kinne (Editor-in-Chief), Oldendorf/Luhe, Germany

\section{LITERATURE CITED}

Aksnes DL, Troedsson C, Thompson EM (2006) Integrating developmental clocking and growth in a life-history model for the planktonic chordate Oikopleura dioica. Mar Ecol Prog Ser 318:81-88

Campbell RG, Wagner MM, Teegarden GJ, Boudreau CA, Durbin EG (2001) Growth and development rates of the copepod Calanus finmarchicus reared in the laboratory. Mar Ecol Prog Ser 221:161-183

Gillooly JF, Charnov EL, West GB, Savage VM, Brown JH (2002) Effects of size and temperature on developmental time. Nature 417:70-73

Ohman MD, Hirche HJ (2001) Density-dependent mortality in an oceanic copepod population. Nature 412: 638-641

Schibler U (2003) Liver regeneration clocks on. Science 302: 234-235

Staver JM, Strathmann RR (2002) Evolution of fast development of planktonic embryos to early swimming. Biol Bull (Woods Hole) 203:58-69

Strathmann RR, Staver RM, Hoffman JR (2002) Risk and the evolution of cell-cycle durations of embryos. Evolution 56: 708-720

Sulston JE, Horvitz HR (1977) Post-embryonic cell lineages of the nematode, Caenorhabditis elegans. Dev Biol 56: $110-156$

Sulston JE, Schierenberg E, White JG, Thomson JN (1983) The embryonic cell lineage of the nematode Caenorhabditis elegans. Dev Biol 100:64-119

Submitted: August 15, 2005; Accepted: January 12, 2006 Proofs received from author(s): July 12, 2006 